\title{
Classification Scheme
}

1. Structure of Crystalline Solids

1.1 Perfectly Periodic Structure

1.2 Solid-State Phase Transformations

1.3 Alloys. Metallurgy

1.4 Microstructure (Magnetic Domains See 18; Ferroelectric Domains See 14.4.1)

1.5 Films

1.6 Surfaces

2. Non-Crystalline State

3. Crystal Growth

4. Bonding Properties

5. Mössbauer Spectroscopy

6. Lattice Dynamics. Phonons

7. Acoustic Properties

8. Thermal Properties

9. Diffusion

10. Defect Properties (Irradiation Defects See 11)

10.1 Metals

10.2 Non-Metals

11. Irradiation Effects (X-Ray Diffraction Investigations See 1 and 10)

12. Mechanical Properties (Plastic Deformations See 10)

12.1 Metals

12.2 Non-Metals

13. Electron States

13.1 Band Structure

13.2 Fermi Surfaces

13.3 Surface and Interface States

13.4 Impurity and Defect States

13.5 Elementary Excitations (Phonons See 6)

13.5.1 Excitons

13.5.2 Plasmons

13.5.3 Polarons

13.5.4 Magnons

14. Electrical Properties. Transport Phenomena

14.1 Metals. Semi-Metals

14.2 Superconductivity. Superconducting Materials and Devices

14.3 Semiconductors

14.3.1 Films

14.3.2 Surfaces and Interfaces

14.3.3 Devices. Junctions (Contact Problems See 14.3.4)

14.3.4 High-Field Phenomena, Space-Charge Effects, Inhomogeneities, Injected

14.4 Dielectrics

Carriers (Electroluminescence See 20.3; Junctions See 14.3.3)

14.4.1 Ferroelectrics

15. Thermoelectric and Thermomagnetic Properties

16. Photoconductivity. Photovoltaic Effects

17. Emission of Electrons and Ions

17.1 Field Emission Microscope Investigations

18. Magnetic Properties

18.1 Paramagnetic Properties

18.2 Ferromagnetic Properties

18.2.1 Ferromagnetic Films

18.3 Ferrimagnetic Properties

18.4 Antiferromagnetic Properties 


\title{
physica status solidi (b)
}

\section{basic research}

\author{
Board of Editors
}

P. AIGRAIN, Paris, S. AMELINCKX, Mol-Donk, V. L. BONCH-BRUEVICH, Moskva, M. CARDONA, Stuttgart,

W.DEK EYSER, Gent, W.FRA NZ, Münster, P.GÖ RLICH, Jena, E. GRILLOT, Paris, E. GUTSCHE, Berlin, R. KAISCHEW, Sofia, P. T. LANDSBERG, Southampton,

S. LUNDQVIST, Göteborg, L. NEEL, Grenoble, A. PIEKARA, Warszawa,

A. SEEGER, Stuttgart, F. SEITZ, New York, O. STASI W, Berlin, M. STEENBECK, Jena, F. STÖCKMANN, Karlsruhe, J. TAUC, Providence

\author{
Editor-in-Chief \\ P. GÖRLICH
}

Advisory Board

M. BALKANSKI, Paris, P. C. BANBURY, Reading, M. BERNARD, Bagneux, W. BRAUER, Berlin, W. COCHRAN, Edinburgh, R. COELHO, Fontenay-aux-Roses, H.-D. DIETZE, Aachen, J. D. ESHELBY, Sheffield, P. P. FEOFILOV, Leningrad,

G. JACOBS, Gent, E. KROENER, Stuttgart, R. KUBO, Tokyo,

M. MATYÁŠ, Praha, H. D. MEGAW, Cambridge, T. S. MOSS, Farnborough,

E. NAGY, Budapest, E. A. NIEK ISCH, Jülich, L. PA L, Budapest, M. RODOT, Bellevue/Seine, H.M. ROSENBERG, Oxford, S.SHIONOYA, Tokyo, W. STEINMANN, München, R. VAUTIER, Bellevue/Seine

Volume $61 \cdot$ Number $1 \cdot$ Pages 1 to $374, \mathrm{~K} 1$ to K52, and A1 to A8

January 1, 1974

PSSB 61(1) 1-374, K1-K52, A1-A8 (1974)

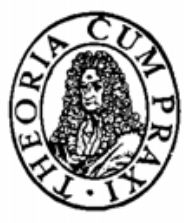

A K A D E M I E - VERLA G • B E R L IN 
Subscriptions and orders for single copies should be addressed to

AKADEMIE-VERLAG, 108 Berlin, Leipziger Straße $3-4$ or to Buchhandlung KUNST UND WISSEN, Erich Bieber, 7 Stuttgart 1, Wilhelmstr. 4-6 or to Deutsche Buch-Export und- Import GmbH, 701 Leipzig, Postschließfach 160

\section{Editorial Note:}

"physica status solidi (b)" undertakes that an original paper accepted for publication before the 8th of any month will be published within 50 days of this date unless the author requests a postponement. In special cases there may be some delay between receipt and acceptance of a paper due to the review and, if necessary, revision of the paper.

Schriftleiter und verantwortlich für den Inhalt: Professor Dr. Dr, h. c. P. Görlich, 102 Berlin, Neue Schönhauser Str. 20 baw. 69 Jena, Humboldtstr. 26. Chefredakteur: Dr. H.-J. Hänsch. Redaktionskollegium: Prof. Dr. E. Gutsche, Dr. H.-J. Hănsch, Dr. H. Lange, Dr. S. Oberländer. Anschrift der Schriftleitung: 102 Berlin, Neue Schönhauser Str. 20, Fernruf: 4223380. Verlag: Akademie-Verlag, 108 Berlin, Leipziger Str. 3-4, Fernruf: 220441, TelexNr. 112020, Postscheckkonto: Berlin 35021. - Die Zeitschrift ,physica status solidi (b)“" erscheint jeweils am 1. des Monats. Bezugspreis eines Bandes 140,- M; Sonderpreis für die DDR 120, - M. Bestellnummer dieses Bandes: $1068 / 61$. Jeder Band enthält zwei Hefte. Gesamtherstellung: VEB Druckerei "Thomas Müntzer" Bad Langensalza. Veröffentlicht unter der Lizenznummer 1310 des Presseamtes beim Vorsitzenden des Ministerrates der Deutschen Demokratischen Republik. Printed in the German Democratic Republic. 